\title{
Dopamine Regulates Two Classes of Primate Prefrontal Neurons That Represent Sensory Signals
}

\author{
Simon N. Jacob, ${ }^{\star}$ Torben Ott, ${ }^{\star}$ and Andreas Nieder \\ Animal Physiology, Institute of Neurobiology, University of Tübingen, D-72076 Tübingen, Germany
}

The lateral prefrontal cortex (PFC), a hub of higher-level cognitive processing, is strongly modulated by midbrain dopamine (DA) neurons. The cellular mechanisms have been comprehensively studied in the context of short-term memory, but little is known about how DA regulates sensory inputs to PFC that precede and give rise to such memory activity. By preparing recipient cortical circuits for incoming signals, DA could be a powerful determinant of downstream cognitive processing. Here, we tested the hypothesis that prefrontal DA regulates the representation of sensory signals that are required for perceptual decisions. In rhesus monkeys trained to report the presence or absence of visual stimuli at varying levels of contrast, we simultaneously recorded extracellular single-unit activity and applied DA to the immediate vicinity of the neurons by micro-iontophoresis. We found that DA modulation of prefrontal neurons is not uniform but tailored to specialized neuronal classes. In one population of neurons, DA suppressed activity with high temporal precision but preserved signal/noise ratio. Neurons in this group had short visual response latencies and comprised all recorded narrow-spiking, putative interneurons. In a distinct population, DA increased excitability and enhanced signal/noise ratio by reducing response variability. These neurons had longer visual response latencies and were composed exclusively of broad-spiking, putative pyramidal neurons. By gating sensory inputs to PFC and subsequently strengthening the representation of sensory signals, DA might play an important role in shaping how the PFC initiates appropriate behavior in response to changes in the sensory environment.

\section{Introduction}

All neuronal systems are subject to neuromodulation, which can profoundly alter the properties of target circuits (Marder, 2012). The primate lateral prefrontal cortex (PFC), a hub of higher-level cognitive functioning (Fuster, 2008; Bongard and Nieder, 2010; Eiselt and Nieder, 2013), receives particularly strong projections from dopamine (DA) neurons in the midbrain (Williams and Goldman-Rakic, 1998; Björklund and Dunnett, 2007). DA neurons fire phasic bursts of action potentials with short latencies of $100-150 \mathrm{~ms}$ in response to behaviorally relevant sensory events (Schultz, 1998; Matsumoto and Hikosaka, 2009). Therefore, it has been suggested that DA could prepare its higher-order target areas for the processing of incoming signals (Redgrave and Gurney, 2006; de Lafuente and Romo, 2011). How might DA influence recipient prefrontal neurons to control information relayed to this important cortical structure?

Prefrontal DA regulates many frontal lobe functions, such as set-shifting and behavioral flexibility (Floresco et al., 2006), association learning (Puig and Miller, 2012), and the maintenance of

\footnotetext{
Received Jan. 16, 2013; revised June 10, 2013; accepted July 9, 2013.

Author contributions: S.N.J. and A.N. designed research; S.N.J. and T.0. performed research; S.N.J. and T.0. analyzed data; S.N.J., T.O., and A.N. wrote the paper.

This work was supported by grants from the German Research Foundation [S.N.J. (Grant JA 1999/1-1) and A.N. (Grant NI 618/2-1)]. We thank A. Thiele for technical assistance with electrode fabrication and iontophoresis, K. Merten for help with animal training and data analysis, and S. Hage and C. Zielinski for comments on this manuscript. *S.N.J. and T.0. contributed equally to this work.

The authors declare no competing financial interests.

Correspondence should be addressed to Dr. Simon N. Jacob, Department of Psychiatry and Psychotherapy, Charité Berlin, Charitéplatz 1, 10117 Berlin, Germany. E-mail: simon.jacob@charite.de.

DOI:10.1523/JNEUROSCI.0210-13.2013

Copyright $\odot 2013$ the authors $\quad 0270-6474 / 13 / 3313724-11 \$ 15.00 / 0$
}

stimuli in working memory (Brozoski et al., 1979). Much of what is known about the mechanisms of DA action in PFC stems from electrophysiological studies on memory-related activity, i.e., in the absence of sensory stimulation (Williams and GoldmanRakic, 1995). In rhesus monkeys engaged in a spatial working memory task, PFC neurons active in the delay period of the task showed improved tuning to preferred remembered locations when stimulated with DA receptor agonists (Vijayraghavan et al., 2007). Therefore, it is believed that the principal function of DA in PFC is to strengthen mental representations (Arnsten, 2011).

In contrast, little is known about how DA modulates prefrontal sensory signals that precede and give rise to such sustained activity. Anecdotal evidence indicates that visual stimuli used to cue a target to be remembered are also influenced by DA (Sawaguchi et al., 1990; Williams and Goldman-Rakic, 1995), but quantitative analysis and an in-depth investigation of the cellular mechanisms are lacking. Because phasic DA activity that is timelocked to relevant sensory stimuli seems particularly suited to regulate the representation of these shorter-lived signals, it has been proposed that DA might serve as a gating signal that controls inputs to PFC (Servan-Schreiber et al., 1990; D’Ardenne et al., 2012). By assigning salience to prefrontal sensory inputs, phasic DA could strongly influence subsequent cognitive processing in PFC. Visual signals, for example, are passed through lower-level cortical areas in a feedforward manner and reach the PFC within 100-150 ms (Thorpe and Fabre-Thorpe, 2001). The PFC collects this sensory information to form subjective judgments, such as regarding the presence or absence of sensory stimulation (de Lafuente and Romo, 2006). Recent electrophysiological studies have demonstrated that the physical intensity of tactile and visual 
stimuli is represented in single neurons of the primate PFC alongside their perceived intensity, i.e., the animal's subjective experience of a stimulus (de Lafuente and Romo, 2005; Merten and Nieder, 2012, 2013).

Here, we investigate in trained rhesus monkeys how DA controls the prefrontal representation of such brief sensory stimuli that must be detected by the animals (Merten and Nieder, 2012, 2013). We found that DA strengthens visual signals by modulating activity in two distinct classes of neurons. Our results suggest that prefrontal DA may play an important role in determining how the PFC orchestrates behavioral responses triggered by sensory events.

\section{Materials and Methods}

\section{Surgical procedures}

Two male rhesus monkeys (Macaca mulatta) were implanted with a titanium head post and one recording chamber centered over the principal sulcus of the lateral PFC, anterior to the frontal eye fields (right hemisphere in monkey $\mathrm{H}$, right and left hemispheres consecutively in monkey $\mathrm{M})$. Surgery was conducted using aseptic techniques under general anesthesia. Structural magnetic resonance imaging was performed before implantation to locate anatomical landmarks. All experimental procedures were in accordance with the guidelines for animal experimentation approved by the local authority, the Regierungspräsidium Tübingen.

\section{Behavioral protocol}

Task. The monkeys were trained to report the presence or absence of visual objects flashed at varying contrast levels centered on their perceptual threshold. The animals initiated each experimental trial by grasping a lever and fixating a central fixation target (fixation period). After 500 $\mathrm{ms}$, a stimulus was displayed for $100 \mathrm{~ms}$ in half of the trials (stimulus period). In the other half, no stimulus was shown. Both trial types were randomly intermixed. After the delay period $(2700 \mathrm{~ms})$, a colored rule cue instructed the monkey how to respond. If a stimulus was presented, a red square cue required the monkey to release the lever within $1000 \mathrm{~ms}$ to receive a fluid reward, whereas a blue cue indicated to the monkey to keep holding the lever for $1200 \mathrm{~ms}$. The rule applied in the inverse way if no stimulus was presented.

CORTEX software (National Institute of Mental Health, Bethesda, MD) was used for experimental control and behavioral data acquisition. The animals maintained fixation throughout the fixation, stimulus, and delay periods within $1.75^{\circ}$ of visual angle of the central fixation target (ISCAN).

Visual stimuli. The stimulus consisted of a gray object ( $4^{\circ}$ of visual angle in diameter) presented at seven levels of contrast close to perceptual threshold, determined individually for each animal (monkey H: 7.3, 8.7, 10.6, 11.6, 19.9, 24.9, and 28.0\%; monkey M: 9.1, 9.8, 11.8, 12.5, 14.7, 16.7 , and $17.4 \%$ ), measured with an LS-100 luminance meter (Konica Minolta). The shape of the object was chosen randomly from a set of two objects: hexagon and circle for monkey $\mathrm{H}$; cross and rhomboid for monkey $\mathrm{M}$. The area of the object was kept constant to maintain the same visual contrast across different shapes.

Visual contrasts were determined for each animal individually to yield approximately the same data points on the psychometric curve. To pool data for analysis, visual contrasts were normalized to an ordinal scale of 1-7 (1 corresponding to the lowest and 7 to the highest stimulus contrast presented to each animal, regardless of the actual physical intensity). Salient stimuli analyzed in Figures 3 and 5 denote the three highest contrasts (5-7).

\section{Electrophysiology}

In each recording session, up to three electrodes (see below, Iontophoresis) were inserted transdurally using a modified electrical microdrive (NAN Instruments). Neurons were recorded at random; no attempt was made to preselect neurons according to particular response properties. Signal acquisition, amplification, filtering, and digitalization were accomplished with the MAP system (Plexon). Waveform separation was performed offline (Offline Sorter; Plexon).

\section{Iontophoresis}

DA was applied iontophoretically (MVCS iontophoresis system; npi electronic) using custom-made tungsten-in-glass electrodes flanked by two pipettes each (Thiele et al., 2006). Electrode impedances were 1-3 $\mathrm{M} \Omega$ (measured at $500 \mathrm{~Hz}$; Omega Tip Z; World Precision Instruments). Pipette resistances depended on the pipette opening diameter, drug, and solvent used. Typical resistances were 15-50 $\mathrm{M} \Omega$ (full range, 15-150 M $\Omega$ ). Pilot in vitro experiments (DA iontophoresis into $\mathrm{NaCl}$, concentrations quantified by HPLC) determined the smallest holding current that ensured good retention without accumulation of dead space and thus allowed for rapid delivery of DA after switching to ejection currents. Retention currents were -7 to $-10 \mathrm{nA}$. Ejection currents for DA ( $200 \mathrm{~mm}$ in double-distilled water, $\mathrm{pH} 4.0$ with $\mathrm{HCl}$; Sigma-Aldrich) were +25-100 $\mathrm{nA}$ (median, $+50 \mathrm{nA}$ ). Control experiments with $0.9 \% \mathrm{NaCl}$, $\mathrm{pH} 7$, used $+50 \mathrm{nA}$. Ejection currents were chosen to match the values reported to be maximally effective, i.e., in the peak range of the inverted-U function (Sawaguchi, 2001; Vijayraghavan et al., 2007). DA currents were varied only during experiments to determine whether the ratio of inhibition/ excitation depended on the applied concentration. Otherwise, we did not attempt to investigate dosage effects.

One pipette per electrode was filled with DA solution, and the other contained $0.9 \% \mathrm{NaCl}$. Electrode impedance and pipette resistance were measured after each recording session. DA was applied continuously for 12-15 min, depending on the number of trials completed correctly by the animal. The first block was always the control condition. Given the fast DA application verified by HPLC (see above), we did not automatically exclude data at the current switching points.

\section{Data analyses}

Data analysis was performed with MATLAB (Mathworks). None of the reported analyses depended on the exact choice of trials to include or time windows to analyze. Repeating analyses with a different choice of parameters yielded comparable results.

Excitability modulation. Neurons stimulated with DA were excluded from additional analysis if their baseline (fixation period) discharge rates were $<1 \mathrm{~Hz}$ in the control or DA phase. Baseline firing rates of each neuron were pooled for the control condition and the DA condition and compared with a rank-sum test (Mann-Whitney $U$ test). If the median firing rate in the DA condition was significantly $(p<0.05$, two-sided test) larger than in the control condition, the neuron was classified as excited, and if the median was lower, the neuron was classified as inhibited by DA.

Receiver operating characteristic analyses. Neuronal coding strength was quantified using receiver operating characteristic (ROC) analysis (Green and Swets, 1966). The area under the ROC curve (auROC) is a nonparametric measure of the discriminability of two distributions. It denotes the probability with which an ideal observer can tell apart a meaningful signal from a noisy background. Values of 0.5 indicate no separation, and values of 1 signal perfect discriminability. The auROC takes into account both the difference between distribution means as well as their widths and is therefore a more suitable indicator of signal quality than other, simpler measures of signal/noise ratio (Servan-Schreiber et al., 1990; Parker and Newsome, 1998; Herrero et al., 2008).

Stimulus-responsive neurons. A two-way ANOVA was calculated with main factors stimulus contrast (salient/absent) and iontophoresis condition (control/DA) using firing rates after stimulus presentation $(300 \mathrm{~ms}$ time window aligned to the individual response latency of the neuron; see below), including correct trials only. Neurons with a significant stimulus main effect $(p<0.05)$ were classified as stimulus responsive. Salient stimulation was defined as the three highest visual contrasts.

Except for the analysis in Figure $7 b$ (see below), visual response latencies were calculated using sliding ROC analysis with a window size of $50 \mathrm{~ms}$, step of $1 \mathrm{~ms}$. For each window, we calculated the auROC by comparing the firing rates between correct salient stimulus trials (hits) and correct absent stimulus trials (correct rejections). To test whether the auROC was significantly different from 0.5 , bootstrapping was used to construct 999 resamples by randomly sampling the data with replacement and maintaining the original number of trials per condition. The latency of a neuron was defined as the time after stimulus onset but no 
later than $500 \mathrm{~ms}$, when the auROC exceeded the $95 \%$ confidence interval of the bootstrapped data for 50 consecutive windows. The response latency was determined separately for the control and DA conditions. If no value could be determined, a default latency corresponding to the median response latency of all neurons in the respective condition was used (228 and $217 \mathrm{~ms}$ for the control and DA conditions, respectively). The choice of these parameters ensured that the analysis window (see below, Neuronal signal metrics) covered the stimulus response in all neurons.

To directly compare visual response latencies between the population of inhibited and excited stimulus encoding neurons (see Fig. $7 b$ ), response latency was defined as two consecutive significant auROC values using a window size of $50 \mathrm{~ms}$, step of $10 \mathrm{~ms}$. This choice of parameters was more sensitive to the actual onset of the stimulus response so that latencies were reliably determined in all stimulus neurons (i.e., no default latencies were used).

For single-cell spike density histograms, the average firing rate in salient trials and trials without visual stimulation (correct trials only) was smoothed with a Gaussian kernel (bin width of $150 \mathrm{~ms}$, step of $1 \mathrm{~ms}$ ). For the population responses, activity was normalized, averaged, and smoothed with the same Gaussian kernel. Responses were normalized by subtracting the mean baseline firing rate in the control condition and dividing by the SD of the baseline firing rates in the control condition.

Stimulus responses calculated using sliding ROC analysis (window size of $300 \mathrm{~ms}$, step of $50 \mathrm{~ms}$ ) quantified the discriminability between the firing rate distributions of correct salient trials and correct rejection trials.

Neuronal signal metrics. All analyses were performed using data from a $300 \mathrm{~ms}$ window aligned to individual visual response latencies. This ensured that stimulus responses were adequately captured in all neurons. To distinguish between additive and multiplicative operations, the difference between the mean firing rate in hit trials and correct rejections was divided by the mean baseline firing rate for all (normalized) contrasts and both iontophoresis conditions (Vijayraghavan et al., 2007). Neurometric curves were determined by calculating the auROC between discharge rates in hit trials and correct rejections for all (normalized) visual contrasts. Neuronal variability was quantified by the Fano factor (FF), i.e., the ratio of trial-by-trial spike count variance and mean spike count (Churchland et al., 2010).

To determine whether DA modulated a signal metric, multiple linear regression analysis was applied to the population data (Merten and Nieder, 2012). Linear functions were fitted to the factors normalized visual contrast and iontophoresis condition (control and DA) using the model for the signal metric $(S): S=a \_0+a \_$stim $\times$STIM $+a \_$ion $\times$ION, where $a \_$stim and $a \_$ion are the coefficients that quantify the signal metric dependence on the normalized stimulus contrast (STIM) and the iontophoresis condition (ION). To assess DA effects on the analyzed signal metric, $p$ values for the factor iontophoresis condition were used ( $t$ statistics for the coefficient $a \_$ion).

DA modulation of neuronal variability was also quantified by multiple linear regression analysis. Linear functions were fitted to the relationship between mean spike count of each contrast and neuron (COUNT) and variance of the spike count of each contrast and neuron (VAR) separately for each iontophoresis condition (ION), i.e., control and DA. An interaction term was included to analyze changes in the slope of the linear functions induced by DA $($ VAR $\times$ ION $)$. The model term was COUNT $=$ $a \_0+a \_$var $\times \mathrm{VAR}+a \_$ion $\times \mathrm{ION}+a \_$int $\times \mathrm{VAR} \times \mathrm{ION}$. $p$ values for the interaction term $a_{-}$int were used to assess DA effects on neuronal variability.

Kinetics of excitability. Exponential functions were fitted to the baseline firing rates of all trials recorded within 6 min of switching to the ejection current (temporal resolution of one trial, i.e., one data point per $5 \mathrm{~s}$ ). Neurons with bad fits (e.g., fitted parameters out of bounds; $n=1$ inhibited cell, $n=4$ excited cells) were excluded from additional analysis. If several DA phases were recorded, baseline firing rates were aligned to all instances of switching to the ejection current and averaged using bins of $5 \mathrm{~s}$. The amplitude of DA modulation was estimated by the mean baseline firing rate in the first or second half of the DA condition for inhibited and excited neurons, respectively. The time course of the base- line firing rate $(\mathrm{FR})$ was expressed as $\mathrm{FR}=\mathrm{A} \times(1-\exp (-x /$ tau $))$, where $A$ is the estimated amplitude and tau the parameter fitted using nonlinear least squares. The population time course was calculated by averaging the normalized baseline discharge rates from all trials recorded within $6 \mathrm{~min}$ before and after switching to DA application using bins of $5 \mathrm{~s}$ and smoothed with a Gaussian kernel (width of $10 \mathrm{~s}$, step of $5 \mathrm{~s}$ ).

Extracellular action potential waveforms. Recorded single units were categorized into narrow-spiking (NS) and broad-spiking (BS) neurons, i.e., putative interneurons and pyramidal cells, using a linear classifier ( $k$-means, $k=2$, squared Euclidean distance) (Diester and Nieder, 2008). For each single unit, the template waveform was extracted with the Plexon Offline sorter. Only neurons with a downward voltage deflection followed by an upward peak were included. Units with a minimum outside $200-400 \mu$ s or a maximum before $300 \mu$ s after reaching the initial threshold were excluded ( $n=3$ of 60 units). Waveforms were normalized by their difference between maximum and minimum voltage deflection and aligned to their minimum. Units in the cluster with the smaller mean spike width constituted the population of NS neurons, and units in the cluster with the larger mean spike width constituted the BS neurons. Interdependence between modulation type (excited or inhibited by DA) and waveform type was tested with Fisher's exact test.

\section{Results}

To determine how DA regulates sensory signals in PFC, we presented brief flashes of visual stimuli at varying contrasts to two rhesus macaque monkeys (Macaca mulatta). The animals were trained to detect the stimuli and report their subjective perceptual judgment about the presence or absence of visual stimulation (Merten and Nieder, 2012) (Fig. 1a). The rule-based task design ensured that neuronal activity in the delay period after the stimulus was free of preparatory motor signals. While the monkeys performed this task, we recorded single units from the lateral PFC. During recordings, trial blocks without pharmacological manipulation (control) alternated with blocks in which DA was applied to the vicinity of the recorded cells by micro-iontophoresis (Fig. 1b). As expected, we did not observe changes in the monkeys' behavior as a consequence of micro-iontophoretic drug application (Fig. 1c,d), because transmitter application with this method is very focal (Herz et al., 1969).

\section{Two classes of DA-sensitive prefrontal neurons}

We recorded 110 neurons that entered the analysis (60 neurons from monkey M, 50 neurons from monkey H). Application of DA influenced the excitability of prefrontal neurons. We compared fixation period activity in the control condition with the DA condition (rank-sum test, $p<0.05$; Fig. $2 a$ ). DA suppressed discharge rates in 32 neurons (DA-inhibited neurons; singleneuron example in Fig. 2b). Activity increased in 28 neurons (DA-excited neurons; single-neuron example in Fig. 2c). Discharge rates were unaffected in 50 neurons (DA-unmodulated neurons; data not shown). The changes in excitability were independent of the iontophoretically applied DA dosage. The proportion of DA-inhibited to DA-excited neurons was not altered when the cell counts were determined separately for lower $(+25-50 \mathrm{nA})$ and higher $(+75-100 \mathrm{nA})$ ejection currents $(23: 22$ versus 9:6, respectively; Fisher's exact test, $p=0.8$ ). None of the physiological parameters analyzed in the following changed in DA-unmodulated cells. This indicates that the effects reported for DA-excited and DA-inhibited neurons were not the result of nonspecific electrical currents.

Inhibitory and excitatory DA effects showed different time courses in the two groups of neurons. In a representative inhibited neuron, DA-mediated suppression of spiking activity was fast and reversed equally rapidly (Fig. $2 b$ ). In a typical excited neuron, DA caused much slower, undulating changes in firing 
a

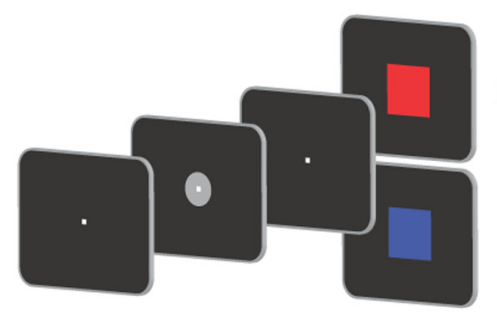

Release

Hold

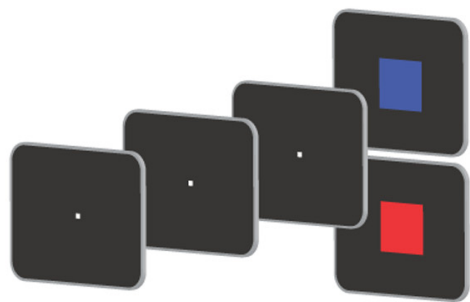

Release

Hold

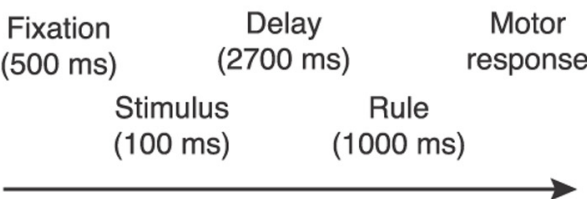

b
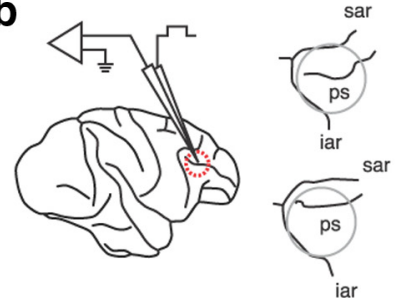

C

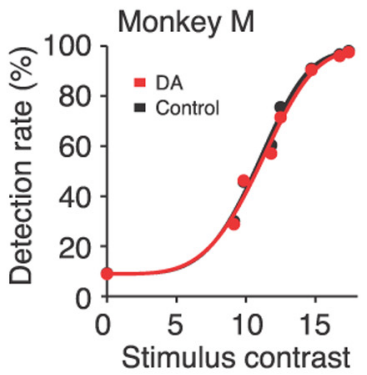

d

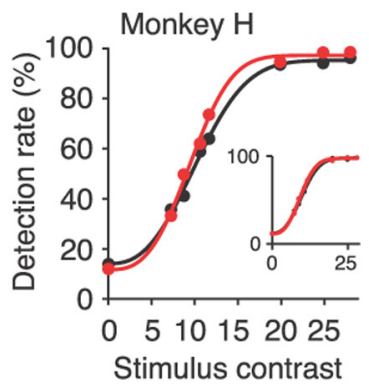

Figure 1. Behavioral protocol and electrophysiological recordings with micro-iontophoresis. $\boldsymbol{a}$, Stimulus detection task requiring the monkeys to report whether a visual stimulus had been presented. A visual stimulus of varying contrast levels was flashed for $100 \mathrm{~ms}$ in $50 \%$ of trials (top). In the other $50 \%$, a blank screen was shown (bottom). $\boldsymbol{b}$, Left, Lateral view of a rhesus monkey brain depicting the location of extracellular neuronal recording and DA iontophoresis in the principal sulcus region of the PFC. Right, Anatomical reconstruction of the recording locations in monkey $\mathrm{M}$ (top) and monkey $\mathrm{H}$ (bottom). $\mathrm{C}$, Psychometric curves with Weibull fits for monkey M ( $n=31$ sessions). Data for control and DA conditions were pooled across sessions. $\boldsymbol{d}$, Conventions as in c for monkey $\mathrm{H}$ ( $n=26$ sessions). The slight difference in performance between control and DA trials in monkey H was attributable to decreased performance at the start of each session ("warm-up" phenomenon; always the control condition) and not the result of DA application. The inset shows psychometric curves for monkey $\mathrm{H}$ with the first $5 \mathrm{~min}(\sim 5 \%)$ of each session omitted. ps, Principal sulcus; sar, superior arcuate sulcus; iar, inferior arcuate sulcus.

rates (Fig. 2c). These effects were confirmed at the population level (Fig. 2d,e): inhibition was precisely time-locked to DA application, whereas DA-mediated excitation reached maximum levels only much later. The rate of change in excitability after DA application was quantified by fitting exponential curves to the temporal profile of neuronal activity. The distribution of time constants suggested categorical differences in the rate of change rather than a gradual transition [mean time constants of $8.9 \pm$ $2.1 \mathrm{~s}$ (median, $3.7 \mathrm{~s}$ ) and $221.9 \pm 37.1 \mathrm{~s}$ (median, $190 \mathrm{~s}$ ) for DA-inhibited and DA-excited neurons, respectively; rank-sum test, $p<0.001$; Figure $2 f, g]$. Control experiments with $\mathrm{NaCl}$ application verified that the rapid reduction in excitability was absent during this sham condition and thus not the result of positive ejection currents ( $n=13$ neurons; Fisher's exact test comparing with DA condition, $p<0.05)$.

\section{Neuron-class-specific modulation of visual responses by DA}

We hypothesized that the categorical changes in excitability might reflect differences in how sensory information is represented in these groups of neurons and how it is modulated by DA. Forty-four percent $(n=14), 36 \%(n=10)$, and $34 \%(n=17)$ of DA-inhibited, DA-excited, and DA-unmodulated neurons, respectively, responded to salient visual stimuli [highest three con- trasts; two-way ANOVA with main effects stimulus (salient/absent) and experimental condition (control/DA), main effect of stimulus, $p<0.05$; Fig. $2 a$ ]. A representative DA-inhibited neuron encoded salient visual stimuli with a clear increase in activity in both the control (Fig. 3a) and DA (Fig. $3 b$ ) conditions. Inhibitory DA effects were reversible and subsided when DA application was discontinued (Fig. $3 c$ ). In contrast, the stimulus response was marginal in an example DA-excited neuron (Fig. $3 d$ ) but increased markedly after DA was applied (Fig. 3e). Again, these changes were clearly reversible (Fig. $3 f$ ).

These single-cell effects were verified at the population level. Across all DAinhibited cells, DA induced an offset in activity but preserved the spike rate difference between trials with salient stimuli and no stimulation (Fig. $3 g, h$ ). However, DA-excited neurons increased stimulus coding based on spike rate differences between trials with salient and absent stimuli (Fig. 3i,j).

We further characterized how DA modulated neuronal excitability. For all contrast levels and neurons, we normalized the stimulus-evoked change in firing rate $\left(\Delta R_{\mathrm{s}}\right.$; difference between mean activity in trials with and without visual stimulation, calculated in a $300 \mathrm{~ms}$ window after stimulus presentation) to baseline activity in the fixation period. Data are presented separately for DA-inhibited and DA-excited neurons in both iontophoresis conditions (Fig. 4a,b). In DAinhibited cells, DA subtracted response levels: the normalized $\Delta R_{\mathrm{s}}$ increased after DA application, i.e., the firing rate difference between trials with and without stimulation was retained at lower baseline firing rates (multiple linear regression, factor iontophoresis condition, $p<0.01$; Fig. $4 a$ ). In DA-excited cells, DA increased gain: there was no change in normalized $\Delta R_{\mathrm{s}}$ with DA, i.e., the firing rate difference increased in proportion to the baseline (multiple linear regression, factor iontophoresis condition, $p=0.5$; Fig. $4 b)$.

\section{Prefrontal DA enhances visual coding strength in excited neurons}

We quantified the capacity of the neurons to discriminate between present and absent visual stimulation, i.e., their coding strength or signal/noise ratio. We compared spike rates in these two conditions by calculating the auROC derived from signal detection theory (Green and Swets, 1966). auROC values of 0.5 indicate no discriminability, and values of 1 indicate signal perfect discriminability. For the representative DA-inhibited neuron from Figure 3, $a$ and $b$, auROC values increased considerably after the presentation of salient stimuli, but they were unaffected by DA application (Fig. $5 a$ ). These time courses were confirmed in the population of DA-inhibited neurons (Fig. 5b). DA did not induce systematic changes in auROC values in this class of cells (seven neurons increased, seven neurons decreased; mean 

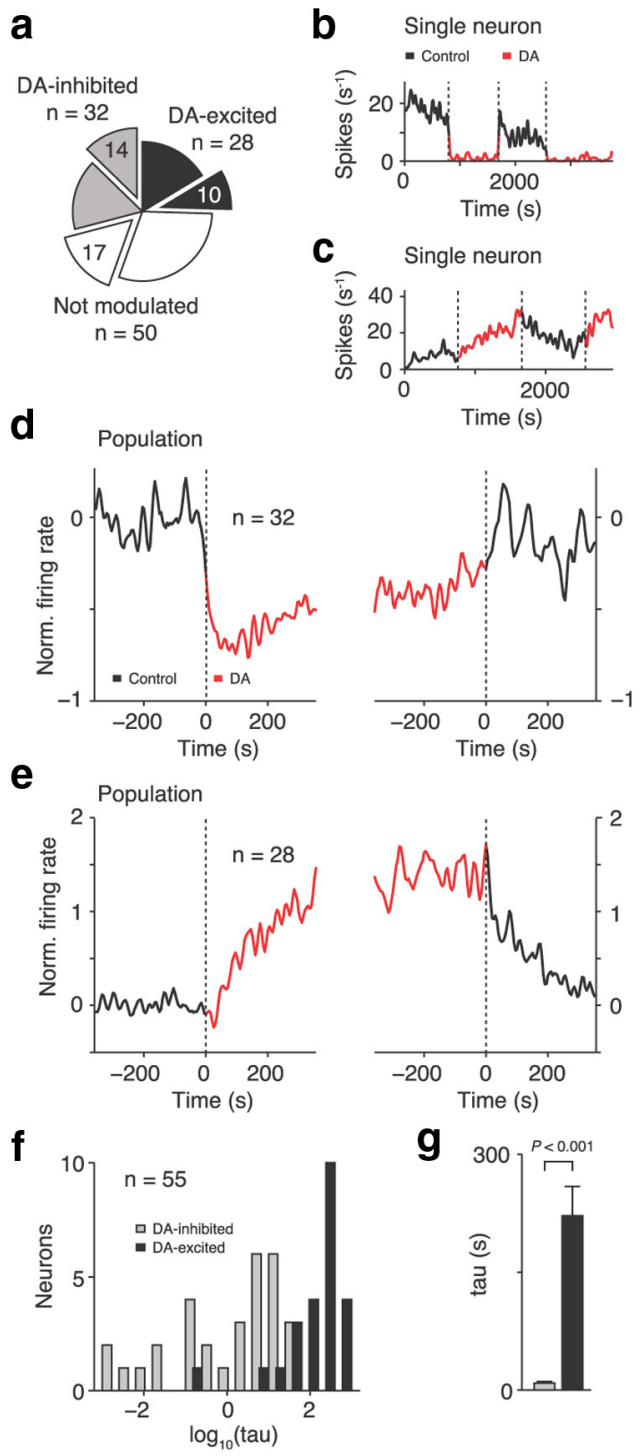

Figure 2. Kinetics of DA modulation in inhibited and excited neurons. $\boldsymbol{a}$, Total number of neurons excited, inhibited, or not modulated by DA together with number of stimulus coding neurons in each group (blown out pie sections). $\boldsymbol{b}-\boldsymbol{e}$, Time courses of responses to DA. Baseline (fixation period) firing rates of an example DA-inhibited $(\boldsymbol{b})$ and DA-excited (c) neuron stimulated repeatedly with $D A$ over the course of $\sim 1 \mathrm{~h}$. Population mean baseline activity of DA-inhibited ( $\boldsymbol{d}$ ) and DA-excited ( $\boldsymbol{e}$ ) neurons aligned to onset (left) and termination (right) of DA application. Inhibition by DA was fast, whereas excitation by DA occurred on longer timescales. $\boldsymbol{f}$, Frequency distribution of time constants (tau) of exponential fits to single-cell data (baseline activity aligned to DA onset). Five neurons with bad fits were excluded and are not shown (see Materials and Methods). $\boldsymbol{g}$, Mean time constants in the two classes of DA-sensitive neurons. Error bars indicate SEM across neurons.

$\Delta$ auROC pooled across contrasts, $-0.0102 \pm 0.0143$; signedrank test, $p=0.5$ ). Mean population auROC values for individual stimuli tended to increase as a function of stimulus contrast and did not change when DA was applied (multiple linear regression; factor contrast, $p=0.12$; factor iontophoresis condition; $p=0.6$; Fig. 5c). Thus, DA did not affect visual coding strength in DA-inhibited neurons.

In contrast, DA significantly improved the stimulus coding quality of DA-excited neurons. Figure $5 d$ shows the time course of the example DA-excited cell from Figure $3, d$ and $e$. Stimulusevoked auROC values increased in this neuron under the influence of DA. This time course was confirmed in the population of DA-excited neurons (Fig. 5e). The increase in auROC values was very consistent across all DA-excited single cells (eight neurons increased, one neuron unchanged, one neuron decreased; mean $\Delta$ auROC pooled across contrasts, $+0.0748 \pm 0.0258$; signedrank test, $p<0.05)$. Mean population auROC values, separated into individual contrasts, increased as a function of stimulus contrast and were significantly higher with DA compared with the control condition (multiple linear regression; factor contrast, $p<0.001$; factor iontophoresis condition; $p<0.001$; Fig. $5 f$ ). No changes were induced by DA in DA-unmodulated neurons (multiple linear regression; factor contrast, $p<0.001$; factor iontophoresis condition; $p=0.14$; data not shown). These results demonstrate that prefrontal DA does not uniformly modify visual coding strength but selectively enhances the capacity to discriminate stimuli from background in the class of DA-excited neurons.

\section{Prefrontal DA reduces neuronal variability in excited neurons}

To investigate which mechanisms could give rise to the strengthening of cortical processing by DA, we determined whether a reduction in neuronal noise (discharge rate variability; Shadlen and Newsome, 1998) might be a contributing factor as hypothesized frequently (Winterer and Weinberger, 2004; Durstewitz and Seamans, 2008; Rolls et al., 2008). To do so, we analyzed the correlation between mean spike counts after stimulus presentation (correct trials) and spike count variance across trials for all neurons in a given class. For quasi-Poisson spiking processes, the data should cluster along the first diagonal (McAdams and Maunsell, 1999). This was the case for DA-inhibited neurons under both control and DA conditions (multiple linear regression, interaction term, $p=0.64$; Fig. $6 a$ ). On a single-cell level, no systematic DA effects on response variability were observed as measured by FF (spike count variance divided by mean; seven neurons increased, seven neurons decreased; mean $\Delta \mathrm{FF}$ pooled across contrasts, $-0.1498 \pm 0.1709$; signed-rank test, $p=0.63$ ). Mean population FFs for each contrast were unchanged in DA-inhibited neurons after application of DA (multiple linear regression; factor iontophoresis condition, $p=0.31$; Fig. $6 b$ ).

In contrast to the findings for DA-inhibited neurons, trial-totrial variability decreased significantly in DA-excited neurons under the influence of DA (multiple linear regression, interaction term, $p<0.01$; Fig. $6 c$ ). The FF reduction was consistent across single cells (eight neurons decreased, two neurons increased; mean $\Delta \mathrm{FF}$ pooled across contrasts, $-0.391 \pm 0.1931$; signedrank test, $p<0.05)$. Mean population FFs, separated into individual contrasts, were significantly reduced by DA compared with the control condition (multiple linear regression, factor iontophoresis condition; $p<0.05$; Fig. $6 d$ ). No changes were induced in DA-unmodulated neurons (multiple linear regression; factor iontophoresis condition; $p=1.0$; data not shown). Thus, DA rendered prefrontal processing more reliable by reducing noise at the level of DA-excited neurons.

\section{Inhibition and excitation control distinct prefrontal processing stages}

To further characterize the two DA-responsive neuron classes, we analyzed the extracellular action potential waveforms of the cells. Electrophysiological recordings have suggested that longer waveforms might be primarily associated with pyramidal cells (BS neurons), whereas shorter waveforms could be more typical of interneurons (NS neurons) (Henze et al., 2000; Diester and Nieder, 2008; Hussar and Pasternak, 2009; Vigneswaran et al., 2011). We calculated the average normalized waveform for each single 
DA-inhibited neurons

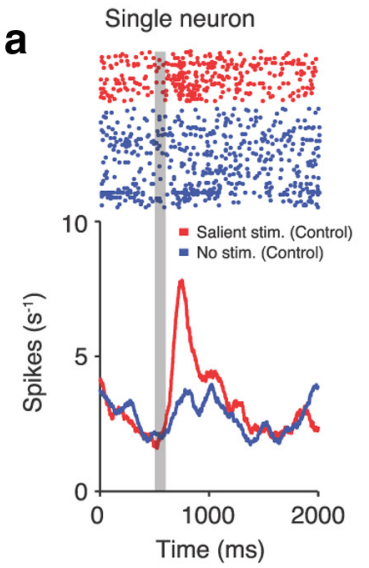

C
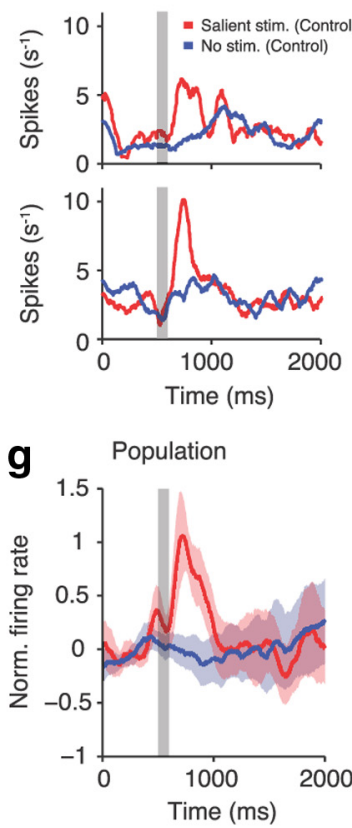

b
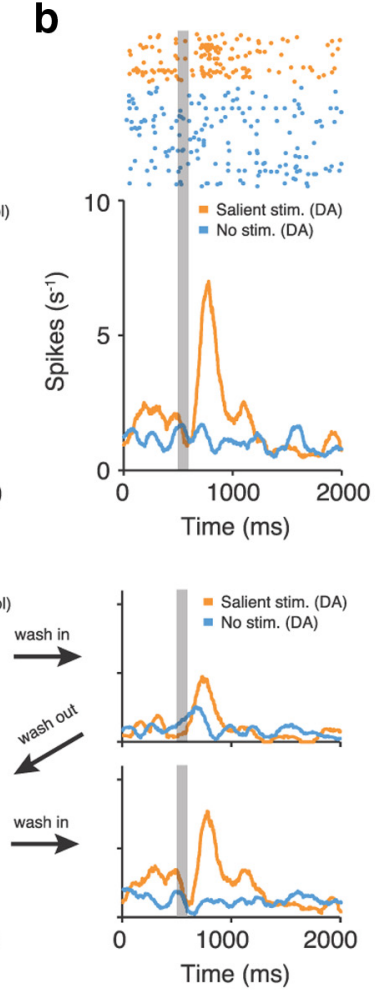

h

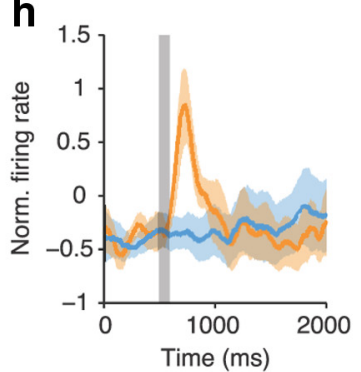

DA-excited neurons
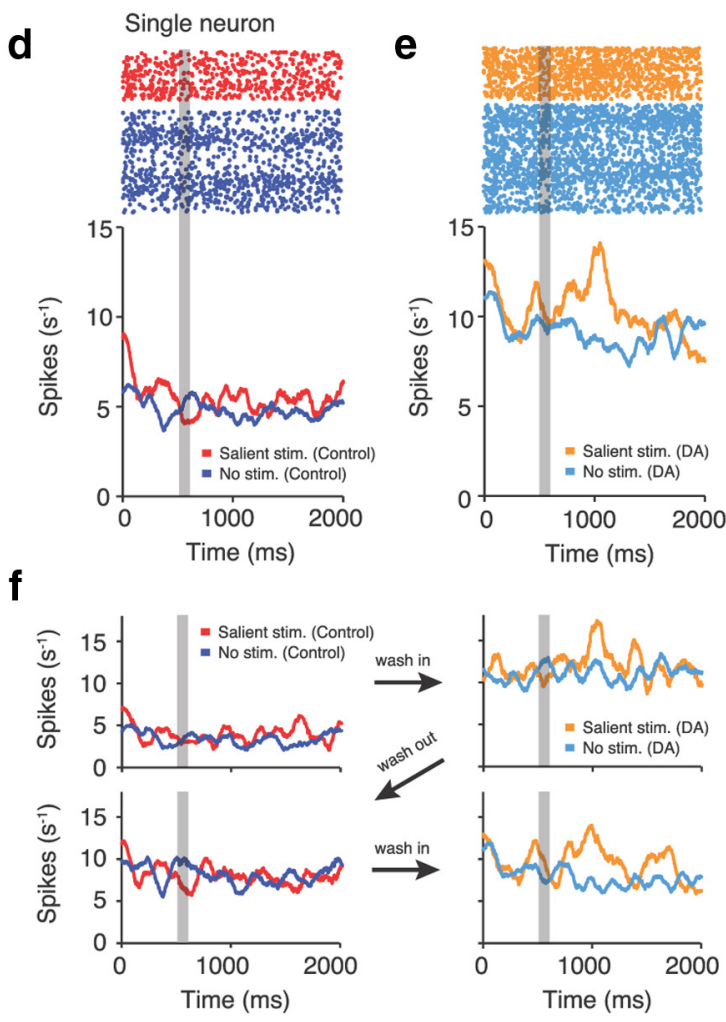

i Population

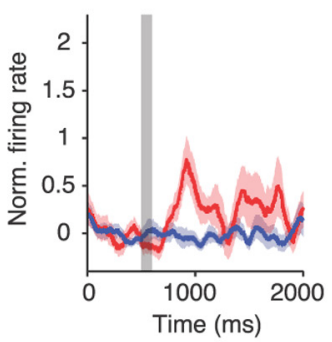

j

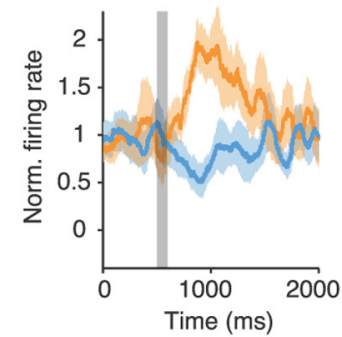

Figure 3. DA modulation of prefrontal visual signals is neuron-class specific. $\boldsymbol{a}, \boldsymbol{b}$, Responses of an example DA-inhibited neuron to salient (highest 3 contrasts) and absent visual stimuli in the control (a) and DA (b) conditions. Activity is aligned to the start of a trial (fixation period). The gray shaded area marks the stimulus presentation. Top, Dot raster plot; bottom, spike density histogram. Visual coding is preserved at shifted response levels. $\boldsymbol{c}$, Sequence of control and DA periods in the same example DA-inhibited neuron. DA-mediated effects are reversible. $\boldsymbol{d}-\boldsymbol{f}$, Conventions as in $\boldsymbol{a}-\boldsymbol{c}$ for an example DA-excited neuron. Visual responses are enhanced by DA. $\boldsymbol{g}, \boldsymbol{h}$, Population mean responses of DA-inhibited neurons in control $(\boldsymbol{g})$ and DA (h) trials. $\boldsymbol{i}, \boldsymbol{j}$, Conventions as in $\boldsymbol{g}$ and $\boldsymbol{h}$, for DA-excited neurons. Shaded areas in $\boldsymbol{g}$ - $\boldsymbol{j}$ indicate SEM across neurons.

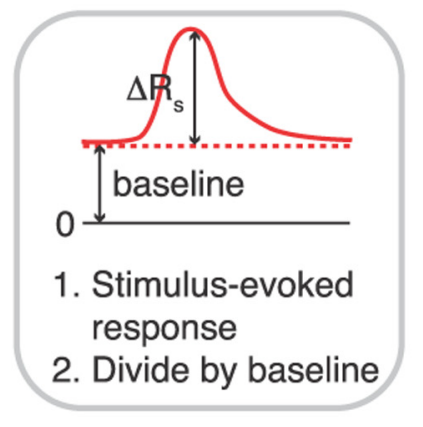

$\mathbf{a}$

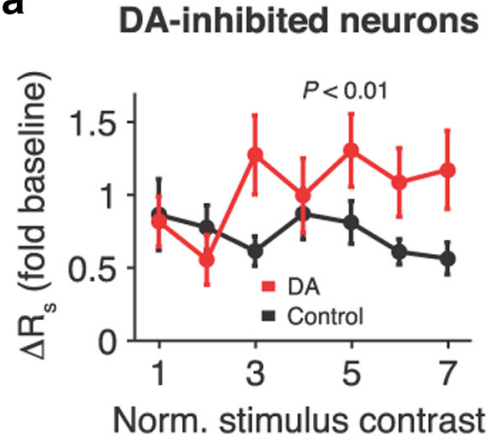

b

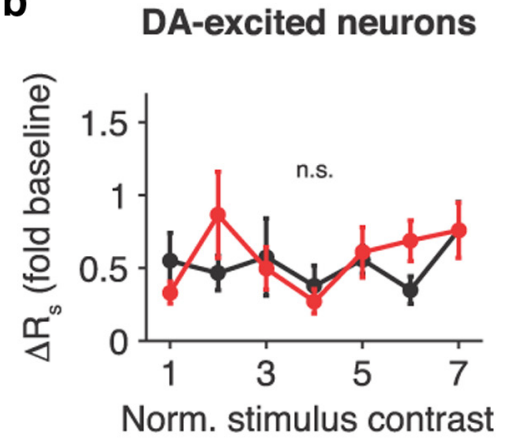

Figure 4. Subtraction and multiplication of activity in DA-inhibited and DA-excited neurons. $\boldsymbol{a}$, Stimulus-evoked change in firing rate normalized to baseline activity in the fixation period, computed as shown by schematic on the left, for DA-inhibited neurons under control and DA conditions. Shifts to larger values indicate that DA offsets activity (additive operation), i.e., the firing rate difference is retained at lower baseline firing rates (subtraction). The animals' perceptual threshold (on the rising slope of the psychometric function; Fig. 1c, $d$ ) corresponds to normalized stimulus contrasts 1-4. $\boldsymbol{b}$, Conventions as in $\boldsymbol{a}$ for DA-excited neurons. Superimposed curves indicate that DA increases gain (multiplicative operation), i.e., the firing rate difference increases in proportion to baseline firing rates. Error bars indicate SEM across neurons. 
neuron and used a linear classifier to objectively separate BS from NS cells. BS and NS waveforms were distributed differently in the classes of DA-excited and DA-inhibited neurons (Fisher's exact test, $p<0.05$; Fig. $7 a$ ). All stimulus-encoding DA-excited cells were BS neurons (putative pyramidal neurons, $n=10$ ). In contrast, there were equal numbers of BS and NS cells (putative interneurons) in the class of stimulus-responsive DA-inhibited neurons $(n=7$ each). Thus, in the group of DA-inhibited neurons, there were more putative interneurons than to be expected by their frequency in neocortex (20-30\%; Markram et al., 2004), and there were significantly more putative pyramidal cells in the class of DA-excited neurons. Interestingly, all stimulus encoding putative interneurons that were responsive to DA were inhibited $(n=7)$. The same pattern was found when all DAresponsive neurons were analyzed (DAexcited neurons: 22 BS, 3 NS; DAinhibited neurons: 17 BS, 15 NS; $p<$ $0.01)$. In accord with the strongly biased distribution of putative interneurons toward DA-inhibited cells, baseline firing rates under control conditions were higher in this group of neurons compared with DA-excited cells, although the difference did not reach significance $(8.3 \pm 1.4$ vs $5.4 \pm 0.8$ spikes/s for DA-inhibited and DA-excited neurons, respectively; ranksum test, $p=0.13$ ). In the instances in which multiple DA-modulated neurons were recorded at the same electrode ( 12 of 45 electrodes), we more often recorded cells from the same class than from different classes (eight vs four electrodes, respectively). These results support the notion that DA-mediated changes in excitability were characteristic of distinct neuronal populations.

We finally explored whether DA-inhibited and DA-excited neurons might be involved at different stages of prefrontal sensory processing. Under control conditions, prefrontal neurons that were inhibited by DA encoded visual signals significantly earlier than DA-excited neurons (mean stimulus response latency, $165 \pm 18$ and $261 \pm 27 \mathrm{~ms}$ for DA-inhibited and DAexcited neurons, respectively; rank-sum test, $p<0.05$; Fig. $7 b$; see also Figs. $3 g, i, 5 b, e)$. DA-inhibited neurons were driven more strongly by sensory input: under control conditions, visual coding strength was higher in this population compared with DAexcited cells across all contrasts (multiple linear regression; factor neuron class, $p<0.001$; Fig. $7 c$ ).

Closer inspection of the population spike density histograms of DA-excited neurons revealed that activity after omission of a stimulus was not a simple continuation of activity in the fixation period when DA had been applied (Fig. 3, compare $i, j$ ). To examine whether DA-excited neurons represented not just physical stimulus intensity but possibly a processing stage more remote from sensory input, we compared baseline activity in the fixation period with firing rates in trials without stimulation, calculated in the same $300 \mathrm{~ms}$ analysis window as previously (Fig. 7d). A deviacross neurons.
DA-inhibited neurons DA-excited neurons

d

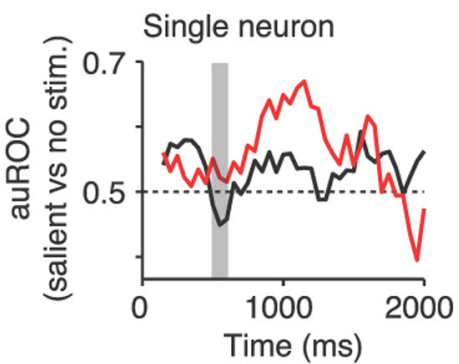

e

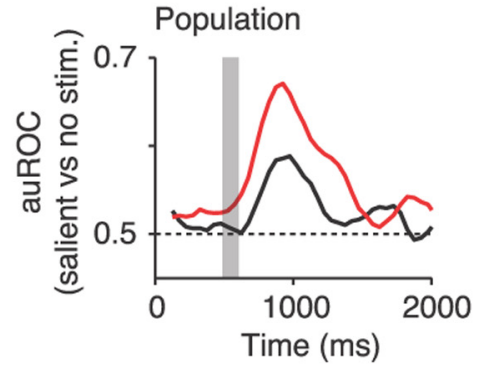

$\mathbf{f}$

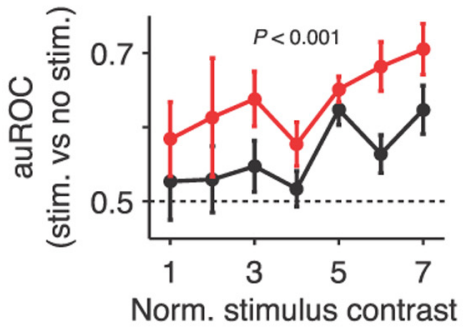

Figure 5. Prefrontal DA enhances visual coding in excited neurons. $\boldsymbol{a}$, Sliding window analysis of visual coding strength (auROC values for salient vs absent visual stimulation) for the example DA-inhibited neuron from Figure $3, a$ and $b$, in correct control and in neurons. $\boldsymbol{d}-\boldsymbol{f}$, Conventions as in $\boldsymbol{a}-\boldsymbol{c}$ for the example DA-excited neuron from Figure $3, \boldsymbol{d}$ and $\boldsymbol{e}(\boldsymbol{d})$ and the population of DA-excited neurons $(\boldsymbol{e}, \boldsymbol{f})$. DA strengthens visual coding in the class of DA-excited neurons. Error bars indicate SEM

ation from zero could suggest that absent stimulation was not encoded as a "default" condition (i.e., a continuation of baseline activity; to be expected for sensory-driven neurons) but instead actively in a potentially more advanced processing step. In DAinhibited neurons, there were no significant differences between baseline activity and activity after the omission of a stimulus in either control or DA conditions (signed-rank test, $p=0.39$ and $p=0.54$, respectively; signed-rank test for difference between control and DA conditions, $p=0.95$; Fig. $7 d$, left). However, in DA-excited neurons, DA application disclosed a deflection from baseline in trials without visual stimulation that was not evident under control conditions (signed-rank test, $p=0.19$ and $p<$ 0.01 , for control and DA conditions, respectively; signed-rank test for difference between control and DA conditions, $p<0.01$; Fig. $7 d$, right). This result suggests that the absence of visual stimulation was represented differently in the two DA-responsive neuron classes.

\section{Discussion}

We report here that DA regulates the representation of sensory information in the primate PFC. We found that prefrontal DA affects two distinct neuronal populations involved in visual coding. DA controlled neurons with short visual response latencies 
DA-inhibited neurons

a

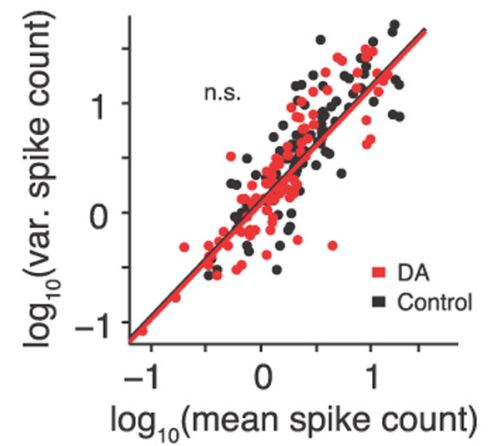

b

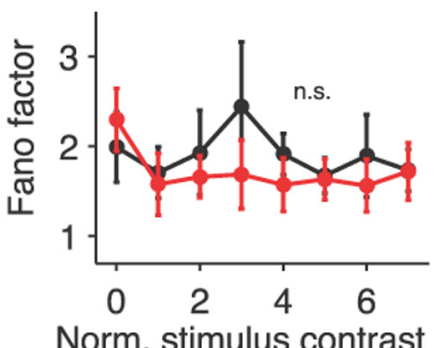

DA-excited neurons

C

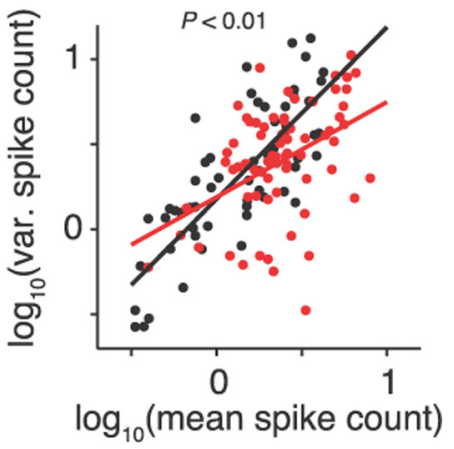

d

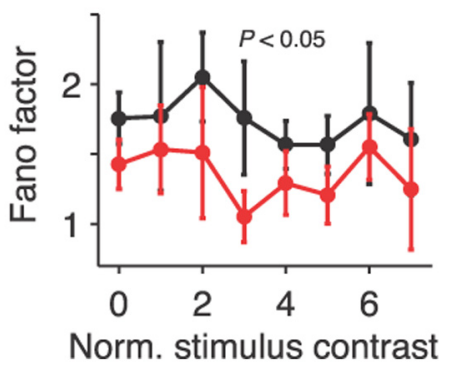

Figure 6. Prefrontal DA reduces response variability in excited neurons. $\boldsymbol{a}$, Mean spike count after stimulus presentation versus spike count variance across trials for DA-inhibited neurons. Each data point represents one neuron and stimulus contrast. Straight lines indicate fits to data. $\boldsymbol{b}$, FFs (spike count variance divided by mean) for all stimulus contrasts in DA-inhibited cells. No changes in response variability are observed after DA application. $\boldsymbol{c}, \boldsymbol{d}$, Conventions as in $\boldsymbol{a}$ and $\boldsymbol{b}$ for DA-excited neurons. The slope of the fitted line is significantly smaller in DA trials compared with control conditions. DA reduces response variability across all contrasts in DA-excited cells. Error bars indicate SEM across neurons.

by suppressing neuronal activity. In neurons with longer response latencies, DA acted as an excitatory modulator and strengthened the representation of visual inputs.

\section{Modes of operation}

Inhibition was implemented principally in the form of a subtractive shift in response levels [additive operation (Silver, 2010); Figs. $3 g, h, 4 a$ ], whereas excitation in the second population resulted from an increase in gain [multiplicative operation (Silver, 2010); Figs. $3 i, j, 4 b]$. In the rodent visual cortex, subtraction is induced by dendrite-targeting interneurons, whereas somatargeting interneurons regulate gain (Wilson et al., 2012). In vitro experiments in the ferret PFC have demonstrated that these classes of interneurons are modulated by DA (Gao et al., 2003). Thus, DA would subtract activity by modulating dendrites and increase gain by controlling the soma (Yang and Seamans, 1996). Our results now suggest that subtraction and multiplication by DA target not the same prefrontal neuron but instead early and late, possibly functionally specialized, processing stages, respectively (Fig. 7).

\section{DA-inhibited neurons}

Control over sensory inputs by inhibition and subtraction of response levels offers a major computational advantage, namely response normalization (Carandini and Heeger, 2012). Inhibitory conductances can adaptively rescale the input of a neuron to match its dynamic range (Mitchell and Silver, 2003) and therefore maximize information transmission (Brenner et al., 2000; Fairhall et al., 2001). Our data indicate that DA afferents to the
PFC might constitute an important pathway to fine-tune and facilitate downstream processing.

DA could also filter distracting, nonpreferred signals by modulating neurotransmission at the dendritic arbor of input layer neurons ("gating") (Durstewitz et al., 2000; Gao et al., 2003). Neurons extracting behaviorally relevant information from a multitude of competing signals would necessarily show temporally precise modulation. Given their rapid responsiveness to DA (Fig. 2f,g), DA-inhibited neurons would be ideal recipients of the phasic signals, e.g., prediction errors, DA neurons relay to the PFC (Redgrave et al., 2008). With a mean stimulus response latency of $165 \mathrm{~ms}$ (Fig. 7b), these cells closely follow the discharge of midbrain DA neurons that typically occurs between 100 and $150 \mathrm{~ms}$ (Dommett et al., 2005; de Lafuente and Romo, 2012). Therefore, DA-inhibited neurons are maximally active at peak extracellular DA concentrations (Schultz, 2007). Thus, DA might reinforce or block signals reaching the PFC and segregate important from distracting information (ServanSchreiber et al., 1990; D’Ardenne et al., 2012). Interestingly, we found that all putative interneurons were inhibited by DA (Fig. 7a). Interneurons are thought to play an important role in the control of information flow in cortex (Constantinidis et al., 2002) and would constitute an ideal target for rapid gating by DA.

At present, the cellular mechanisms by which DA could mediate fast inhibition are unclear (Seamans and Yang, 2004). Inhibitory DA effects are generally reported on longer timescales as a result of technical constraints, such as bath application of dopaminergic drugs. It is also conceivable that the applied DA binds to non-dopaminergic receptors, such as adrenergic receptors, especially at higher concentrations. Iontophoresis is nonquantitative and generally does not provide reliable assessments of the drug concentrations reaching individual neurons. Therefore, additional experiments are required to resolve the issue of pharmacological specificity as well as to determine whether the observed decrease in excitability is indeed the result of phasic, time-locked signaling or generated by longer-lasting mechanisms.

In behaving nonhuman primates, neuronal inhibition has been identified as an important mechanism by which DA affects prefrontal signal processing. DA suppresses neuronal activity in spatially tuned prefrontal neurons engaged in memory-guided saccade tasks and enhances tuning for the remembered saccade target location ("sculpting inhibition"; Williams and GoldmanRakic, 1995; Vijayraghavan et al., 2007; Arnsten, 2011). Because subtraction sharpens stimulus selectivity, i.e., tuning (Wilson et al., 2012), we propose that the spatially tuned cells described previously belong to the class of DA-inhibited neurons identified here. Although the ROC measures we used are well suited for analyzing binary yes-no, e.g., stimulus present-absent decisions (Green and Swets, 1966), we did not detect an increase in signal/ noise ratio as defined by the auROC in DA-inhibited neurons 

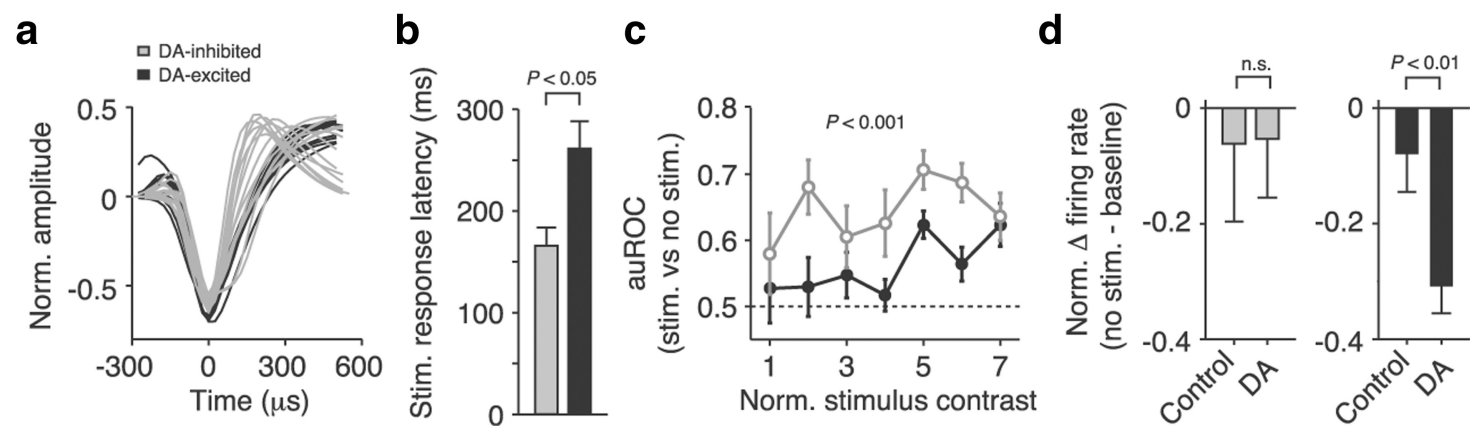

Figure 7. DA modulates distinct prefrontal processing stages. $\boldsymbol{a}$, Normalized average waveforms of stimulus-encoding DA-inhibited and DA-excited neurons. All DA-excited cells were BS neurons, and NS neurons were all inhibited by DA. $\boldsymbol{b}$, Visual response latencies of DA-inhibited and DA-excited neurons under control conditions. DA-inhibited neurons encode visual signals significantly earlier. c, Visual coding strength of DA-inhibited and DA-excited neurons under control conditions (auROC values comparing firing rates between trials with and without visual stimulation). DA-inhibited neurons are driven more strongly by visual stimulation across all contrast levels. $\boldsymbol{d}$, Normalized difference between baseline activity in the fixation period and activity after omission of a stimulus. Firing rates were identical in DA-inhibited neurons in both control and DA trials. In DA-excited neurons, absence of visual stimulation induced a deflection from baseline when DA had been applied. Error bars indicate SEM across neurons.

(Fig. $5 a-c)$. Other response characteristics that are not adequately captured by signal detection theory, e.g., sharpening of tuning curves, might nevertheless create advantages for cortical processing. We also considered the possibility that inhibited neurons were the result of higher intrinsic DA tone and excited neurons were subject to lower DA levels. However, this is unlikely because the ratio of inhibition to excitation was independent of iontophoretic DA dosage, and we did not observe more inhibited neurons at higher DA currents. More experiments tapping different behavioral demands are needed to determine whether the benefits conveyed by DA-induced inhibition lie primarily in rescaling and gating inputs to PFC or whether DA can also affect signal strength per se at this stage.

\section{DA-excited neurons}

In DA-excited neurons, stimulus responses increased in proportion to baseline activity, indicating a multiplicative increase in gain (Servan-Schreiber et al., 1990; Thurley et al., 2008; Figs. 3i,j, $4 b$ ). Although the strength of sensory inputs was unchanged in DA-inhibited neurons, DA selectively increased signal/noise ratio in excited cells (Fig. $5 d-f$ ). In addition, stimuli were encoded more reliably because trial-to-trial variability dropped (Fig. $6 c, d$ ). All three effects closely resemble changes in visual signals observed in visual cortex when attention is allocated in a top-down manner (McAdams and Maunsell, 1999; Mitchell et al., 2007; Noudoost and Moore, 2011a). In other words, prefrontal DA may act as a pharmacological spotlight, directing "attention" toward relevant sensory inputs and enhancing their representation at the level of DA-excited neurons (Brunel and Wang, 2001; Noudoost and Moore, 2011b). Interestingly, DA-induced excitation occurred on considerably longer timescales than inhibition (Fig. $2 f, g)$. Therefore, it is unlikely that the amplification of stimulus coding could be controlled on a trial-by-trial basis in these neurons. DA-excited neurons might not serve the purpose of a flexible, rapidly responsive gate for sensory signals but instead reflect a later processing stage more remote from early sensory inputs. In support of this idea, our analysis of extracellular waveforms did not reveal any putative interneurons in this group of cells but exclusively putative pyramidal neurons (Fig. 7a). Also, DAexcited neurons processed visual inputs almost $100 \mathrm{~ms}$ later than DA-inhibited neurons (Fig. 7b). They were driven less strongly by visual stimuli (Fig. 7c) and encoded absent stimulation actively by a deflection in baseline firing instead of passively as a default condition like DA-inhibited neurons (Fig. 7d). This transient de- pression of activity could reflect, for example, an anticipatory response and contributed to the improved discriminability of stimulus and background in these neurons. In any case, it suggests that DA-excited neurons were not truthful encoders of the physical properties of visual stimuli but might constitute an additional step in the goal-directed evaluation of sensory signals.

Although application of transmitters with micro-iontophoresis is very focal (Herz et al., 1969; Hupé et al., 1999), we cannot exclude that the slower response kinetics in DA-excited neurons were attributable to the fact that DA had to diffuse to a different cortical layer before indirectly taking effect on this class of cells. Another possibility is that DA-excited neurons are modulated not by phasic DA but by tonic transmitter release. Compared with DA neuron bursting, little is known about the function of tonic DA signaling (Floresco et al., 2003). It is thought to reflect increased activity in populations of DA neurons and causes an elevation mainly of extrasynaptic transmitter. Tonic extracellular DA levels do not reach the high levels found in the synaptic cleft and might modulate primarily high-affinity extrasynaptic DA receptors on presynaptic terminals (Grace et al., 2007). We presently do not know the cellular receptors that are involved in generating the effects reported here. Therefore, additional studies will have to address whether DA-excited neurons differ from DA-inhibited cells, for example, in their modulation by the two DA receptor families found in PFC, the $D_{1} R$ and $D_{2} R$, or other catecholamine receptors (Seamans and Yang, 2004; Wang et al., 2004; Noudoost and Moore, 2011a). Differences in the cellular and molecular composition of prefrontal DA-sensitive neurons could allow for targeted modulation of specific cortical signals by DA (Noudoost and Moore, 2011b). For example, in the frontal eye fields, behavioral effects of DA on attentional processing depend on whether injections were made in supragranular or infragranular layers that are characterized by distinct DA receptor profiles (Noudoost and Moore, 2011a). We now find that DAsensitive neurons in more anterior lateral PFC are heterogeneous with regard to the sensory information they carry and how they are modulated by DA. Adding to previous studies, our experiments suggest that the timing and strength of DA neurotransmission could have a strong influence on how this modulatory signal is received and processed in PFC.

\section{Implications for mental diseases}

DA is strongly linked to neuropsychiatric diseases that involve the frontal lobes, such as attention-deficit hyperactivity disorder or 
schizophrenia (Arnsten, 2011). By strengthening sensory inputs, prefrontal DA could be a critical factor in resolving ambiguous sensory events or maintaining the focus of attention. It is tempting to speculate that the observed DA effects could help safeguard the healthy mind, e.g., from hallucinations and intrusions of thought that are characteristic of these mental diseases (Winterer and Weinberger, 2004; Rolls et al., 2008; Fletcher and Frith, 2009). For example, it is frequently hypothesized that the symptom relief conveyed by antipsychotic drugs targeting the DA system, in particular the $D_{2} R$, results from the fact that they decrease noise in prefrontal circuits (Winterer and Weinberger, 2004; Rolls et al., 2008). Our experiments now provide evidence on a cellular level that DA indeed controls neuronal variability in the primate brain.

In conclusion, we have demonstrated that DA neuromodulation in PFC is not uniform but tailored to functionally specialized neurons in the prefrontal processing stream (Arnsten et al., 2012). By controlling sensory inputs to the PFC, DA could be a powerful determinant of how the primate brain uses these signals to generate intelligent behavior in interactions with its sensory environment.

\section{References}

Arnsten AF (2011) Catecholamine influences on dorsolateral prefrontal cortical networks. Biol Psychiatry 69:e89-e99. CrossRef Medline

Arnsten AF, Wang MJ, Paspalas CD (2012) Neuromodulation of thought: flexibilities and vulnerabilities in prefrontal cortical network synapses. Neuron 76:223-239. CrossRef Medline

Björklund A, Dunnett SB (2007) Dopamine neuron systems in the brain: an update. Trends Neurosci 30:194-202. CrossRef Medline

Bongard S, Nieder A (2010) Basic mathematical rules are encoded by primate prefrontal cortex neurons. Proc Natl Acad Sci U S A 107:2277-2282. CrossRef Medline

Brenner N, Bialek W, de Ruyter van Steveninck R (2000) Adaptive rescaling maximizes information transmission. Neuron 26:695-702. CrossRef Medline

Brozoski TJ, Brown RM, Rosvold HE, Goldman PS (1979) Cognitive deficit caused by regional depletion of dopamine in prefrontal cortex of rhesus monkey. Science 205:929-932. CrossRef Medline

Brunel N, Wang XJ (2001) Effects of neuromodulation in a cortical network model of object working memory dominated by recurrent inhibition. J Comput Neurosci 11:63-85. CrossRef Medline

Carandini M, Heeger DJ (2012) Normalization as a canonical neural computation. Nat Rev Neurosci 13:51-62. CrossRef Medline

Churchland MM, Yu BM, Cunningham JP, Sugrue LP, Cohen MR, Corrado GS, Newsome WT, Clark AM, Hosseini P, Scott BB, Bradley DC, Smith MA, Kohn A, Movshon JA, Armstrong KM, Moore T, Chang SW, Snyder LH, Lisberger SG, Priebe NJ, et al. (2010) Stimulus onset quenches neural variability: a widespread cortical phenomenon. Nat Neurosci 13:369378. CrossRef Medline

Constantinidis C, Williams GV, Goldman-Rakic PS (2002) A role for inhibition in shaping the temporal flow of information in prefrontal cortex. Nat Neurosci 5:175-180. CrossRef Medline

D’Ardenne K, Eshel N, Luka J, Lenartowicz A, Nystrom LE, Cohen JD (2012) Role of prefrontal cortex and the midbrain dopamine system in working memory updating. Proc Natl Acad Sci U S A 109:19900-19909. CrossRef Medline

de Lafuente V, Romo R (2005) Neuronal correlates of subjective sensory experience. Nat Neurosci 8:1698-1703. CrossRef Medline

de Lafuente V, Romo R (2006) Neural correlate of subjective sensory experience gradually builds up across cortical areas. Proc Natl Acad Sci U S A 103:14266-14271. CrossRef Medline

de Lafuente V, Romo R (2011) Dopamine neurons code subjective sensory experience and uncertainty of perceptual decisions. Proc Natl Acad Sci U S A 108:19767-19771. CrossRef Medline

de Lafuente V, Romo R (2012) Dopaminergic activity coincides with stimulus detection by the frontal lobe. Neuroscience 218:181-184. CrossRef Medline

Diester I, Nieder A (2008) Complementary contributions of prefrontal neuron classes in abstract numerical categorization. J Neurosci 28: 7737-7747. CrossRef Medline

Dommett E, Coizet V, Blaha CD, Martindale J, Lefebvre V, Walton N, Mayhew JE, Overton PG, Redgrave P (2005) How visual stimuli activate dopaminergic neurons at short latency. Science 307:1476-1479. CrossRef Medline

Durstewitz D, Seamans JK (2008) The dual-state theory of prefrontal cortex dopamine function with relevance to catechol-o-methyltransferase genotypes and schizophrenia. Biol Psychiatry 64:739-749. CrossRef Medline

Durstewitz D, Seamans JK, Sejnowski TJ (2000) Dopamine-mediated stabilization of delay-period activity in a network model of prefrontal cortex. J Neurophysiol 83:1733-1750. Medline

Eiselt AK, Nieder A (2013) Representation of abstract quantitative rules applied to spatial and numerical magnitudes in primate prefrontal cortex. J Neurosci 33:7526-7534. CrossRef Medline

Fairhall AL, Lewen GD, Bialek W, de Ruyter Van Steveninck RR (2001) Efficiency and ambiguity in an adaptive neural code. Nature 412:787-792. CrossRef Medline

Fletcher PC, Frith CD (2009) Perceiving is believing: a Bayesian approach to explaining the positive symptoms of schizophrenia. Nat Rev Neurosci 10:48-58. CrossRef Medline

Floresco SB, West AR, Ash B, Moore H, Grace AA (2003) Afferent modulation of dopamine neuron firing differentially regulates tonic and phasic dopamine transmission. Nat Neurosci 6:968-973. CrossRef Medline

Floresco SB, Magyar O, Ghods-Sharifi S, Vexelman C, Tse MT (2006) Multiple dopamine receptor subtypes in the medial prefrontal cortex of the rat regulate set-shifting. Neuropsychopharmacology 31:297-309. CrossRef Medline

Fuster JM (2008) The prefrontal cortex, Ed 4. London: Academic.

Gao WJ, Wang Y, Goldman-Rakic PS (2003) Dopamine modulation of perisomatic and peridendritic inhibition in prefrontal cortex. J Neurosci 23:1622-1630. Medline

Grace AA, Floresco SB, Goto Y, Lodge DJ (2007) Regulation of firing of dopaminergic neurons and control of goal-directed behaviors. Trends Neurosci 30:220-227. CrossRef Medline

Green DM, Swets JA (1966) Signal detection theory and psychophysics. New York: Wiley.

Henze DA, Borhegyi Z, Csicsvari J, Mamiya A, Harris KD, Buzsáki G (2000) Intracellular features predicted by extracellular recordings in the hippocampus in vivo. J Neurophysiol 84:390-400. Medline

Herrero JL, Roberts MJ, Delicato LS, Gieselmann MA, Dayan P, Thiele A (2008) Acetylcholine contributes through muscarinic receptors to attentional modulation in V1. Nature 454:1110-1114. CrossRef Medline

Herz A, Zieglgänsberger W, Färber G (1969) Microelectrophoretic studies concerning the spread of glutamic acid and GABA in brain tissue. Exp Brain Res 9:221-235. Medline

Hupé JM, Chouvet G, Bullier J (1999) Spatial and temporal parameters of cortical inactivation by GABA. J Neurosci Methods 86:129-143. CrossRef Medline

Hussar CR, Pasternak T (2009) Flexibility of sensory representations in prefrontal cortex depends on cell type. Neuron 64:730-743. CrossRef Medline

Marder E (2012) Neuromodulation of neuronal circuits: back to the future. Neuron 76:1-11. CrossRef Medline

Markram H, Toledo-Rodriguez M, Wang Y, Gupta A, Silberberg G, Wu C (2004) Interneurons of the neocortical inhibitory system. Nat Rev Neurosci 5:793-807. CrossRef Medline

Matsumoto M, Hikosaka O (2009) Two types of dopamine neuron distinctly convey positive and negative motivational signals. Nature 459 : 837-841. CrossRef Medline

McAdams CJ, Maunsell JH (1999) Effects of attention on the reliability of individual neurons in monkey visual cortex. Neuron 23:765-773. CrossRef Medline

Merten K, Nieder A (2012) Active encoding of decisions about stimulus absence in primate prefrontal cortex neurons. Proc Natl Acad Sci U S A 109:6289-6294. CrossRef Medline

Merten K, Nieder A (2013) Comparison of abstract decision encoding in the monkey prefrontal cortex, the presupplementary and cingulate motor areas. J Neurophysiol 110:19-32. CrossRef Medline

Mitchell JF, Sundberg KA, Reynolds JH (2007) Differential attentiondependent response modulation across cell classes in macaque visual area V4. Neuron 55:131-141. CrossRef Medline 
Mitchell SJ, Silver RA (2003) Shunting inhibition modulates neuronal gain during synaptic excitation. Neuron 38:433-445. CrossRef Medline

Noudoost B, Moore T (2011a) Control of visual cortical signals by prefrontal dopamine. Nature 474:372-375. CrossRef Medline

Noudoost B, Moore T (2011b) The role of neuromodulators in selective attention. Trends Cogn Sci 15:585-591. CrossRef Medline

Parker AJ, Newsome WT (1998) Sense and the single neuron: probing the physiology of perception. Annu Rev Neurosci 21:227-277. CrossRef Medline

Puig MV, Miller EK (2012) The role of prefrontal dopamine D1 receptors in the neural mechanisms of associative learning. Neuron 74:874-886. CrossRef Medline

Redgrave P, Gurney K (2006) The short-latency dopamine signal: a role in discovering novel actions? Nat Rev Neurosci 7:967-975. CrossRef Medline

Redgrave P, Gurney K, Reynolds J (2008) What is reinforced by phasic dopamine signals? Brain Res Rev 58:322-339. CrossRef Medline

Rolls ET, Loh M, Deco G, Winterer G (2008) Computational models of schizophrenia and dopamine modulation in the prefrontal cortex. Nat Rev Neurosci 9:696-709. CrossRef Medline

Sawaguchi T (2001) The effects of dopamine and its antagonists on directional delay-period activity of prefrontal neurons in monkeys during an oculomotor delayed-response task. Neurosci Res 41:115-128. CrossRef Medline

Sawaguchi T, Matsumura M, Kubota K (1990) Catecholaminergic effects on neuronal activity related to a delayed response task in monkey prefrontal cortex. J Neurophysiol 63:1385-1400. Medline

Schultz W (1998) Predictive reward signal of dopamine neurons. J Neurophysiol 80:1-27. Medline

Schultz W (2007) Multiple dopamine functions at different time courses. Annu Rev Neurosci 30:259-288. CrossRef Medline

Seamans JK, Yang CR (2004) The principal features and mechanisms of dopamine modulation in the prefrontal cortex. Prog Neurobiol 74:1-58. CrossRef Medline

Servan-Schreiber D, Printz H, Cohen JD (1990) A network model of catecholamine effects: gain, signal-to-noise ratio, and behavior. Science 249: 892-895. CrossRef Medline

Shadlen MN, Newsome WT (1998) The variable discharge of cortical neu- rons: implications for connectivity, computation, and information coding. J Neurosci 18:3870-3896. Medline

Silver RA (2010) Neuronal arithmetic. Nat Rev Neurosci 11:474-489. CrossRef Medline

Thiele A, Delicato LS, Roberts MJ, Gieselmann MA (2006) A novel electrode-pipette design for simultaneous recording of extracellular spikes and iontophoretic drug application in awake behaving monkeys. J Neurosci Methods 158:207-211. CrossRef Medline

Thorpe SJ, Fabre-Thorpe M (2001) Seeking categories in the brain. Science 291:260-263. CrossRef Medline

Thurley K, Senn W, Lüscher HR (2008) Dopamine increases the gain of the input-output response of rat prefrontal pyramidal neurons. J Neurophysiol 99:2985-2997. CrossRef Medline

Vigneswaran G, Kraskov A, Lemon RN (2011) Large identified pyramidal cells in macaque motor and premotor cortex exhibit "thin spikes": implications for cell type classification. J Neurosci 31:14235-14242. CrossRef Medline

Vijayraghavan S, Wang M, Birnbaum SG, Williams GV, Arnsten AF (2007) Inverted- $\mathrm{U}$ dopamine $\mathrm{D} 1$ receptor actions on prefrontal neurons engaged in working memory. Nat Neurosci 10:376-384. CrossRef Medline

Wang M, Vijayraghavan S, Goldman-Rakic PS (2004) Selective D2 receptor actions on the functional circuitry of working memory. Science 303:853-856. CrossRef Medline

Williams GV, Goldman-Rakic PS (1995) Modulation of memory fields by dopamine D1 receptors in prefrontal cortex. Nature 376:572-575. CrossRef Medline

Williams SM, Goldman-Rakic PS (1998) Widespread origin of the primate mesofrontal dopamine system. Cereb Cortex 8:321-345. CrossRef Medline

Wilson NR, Runyan CA, Wang FL, Sur M (2012) Division and subtraction by distinct cortical inhibitory networks in vivo. Nature 488:343-348. CrossRef Medline

Winterer G, Weinberger DR (2004) Genes, dopamine and cortical signalto-noise ratio in schizophrenia. Trends Neurosci 27:683-690. CrossRef Medline

Yang CR, Seamans JK (1996) Dopamine D1 receptor actions in layers V-VI rat prefrontal cortex neurons in vitro: modulation of dendritic-somatic signal integration. J Neurosci 16:1922-1935. Medline 